\title{
NONCOMMUTATIVE ALGEBRAS OF DIMENSION THREE OVER INTEGRAL SCHEMES
}

\author{
BY
}

RICK MIRANDA AND MINA TEICHER

\begin{abstract}
In this article we describe the algebraic data which is equivalent to giving an associative, noncommutative algebra $O_{X}$ over an integral $k$-scheme $Y$ (where $k$ is an algebraically closed field of characteristic $\neq 3$ ), which is locally free of rank 3. The description allows us to conclude that, essentially, all such are locally upper triangular $2 \times 2$ matrices, with degenerations of a restricted form allowed.
\end{abstract}

0. Introduction. Let $k$ be an algebraically closed field of characteristic unequal to 3 , and let $Y$ be an integral $k$-scheme. In this article we will describe the data necessary and sufficient to construct a noncommutative $O_{Y}$-algebra $O_{X}$ which is associative with identity, and which is a locally free of rank 3 as an $O_{Y}$-module.

The "obvious" construction for such an algebra is to take locally free $O_{Y}$-module $F$ of rank 2, and a nowhere zero section $s: O_{Y} \rightarrow F$. The subbundle $L$ of $F$ generated by $s$ will be of rank 1 , and the algebra $O_{X}$ of endomorphisms of $F$ preserving $L$ will be locally free of rank 3 over $O_{Y}$; locally, $O_{X}$ is isomorphic to the algebra of upper triangular $2 \times 2$ matrices. Our main theorem is that all noncommutative algebras of rank 3 are obtained using a similar construction, where the section $s$ is allowed to have zeros; a precise statement is given in Theorem 10.

The method used to analyze these algebras is similar to that employed in $[\mathbf{1}]$, where the commutative case was studied, and some algebro-geometric applications were made. It is somewhat surprising that the answer in the noncommutative case is much simpler than in the commutative case.

The first author would like to thank the NSF and Bar-Ilan University for their generous support while doing this research.

1. The local analysis. Let $O_{X}$ be a noncommutative $O_{Y}$-algebra of rank 3 . Since char $k \neq 3$, the natural inclusion of $O_{Y}$ into $O_{X}$ is split by one-third of the trace map. Let $E \subset O_{X}$ be the locally free rank 2 submodule of $O_{X}$ consisting (locally) of those elements whose trace is zero; in this case we have $O_{X} \cong O_{Y} \oplus E$ as $O_{Y}$-modules. The multiplication in $O_{X}$ is an $O_{Y}$-linear map $O_{X} \otimes O_{Y} O_{X} \rightarrow O_{X}$, and is induced from the multiplication $\phi: E \otimes \mathcal{O}_{Y} E \rightarrow O_{X} \cong O_{Y} \oplus E$ of elements of $E$; the other factors of the multiplication in $O_{X}$ are the natural multiplication in $O_{Y}$ and the left and right $O_{Y}$-module structure on $E$. We are thus naturally led to the following question: What properties does the map $\phi$ enjoy in this situation? Conversely, we can ask: Which maps $\phi$ induce a noncommutative associative multiplication on $\mathrm{O}_{Y} \oplus E$ for which $E$ is the "trace zero" submodule? The answer, locally, is given by the following

Received by the editors April 23, 1984.

1980 Mathematics Subject Classification. Primary 16A46, 16A48. 
Proposition 1. Let $O_{Y}$ be a local integral domain of finite type over $k, E$ a free rank $2 O_{Y}$-module, and $\phi: E \otimes \mathcal{O}_{Y} E \rightarrow O_{Y} \oplus E$ an $O_{Y}$-linear map. Let $O_{X}=O_{Y} \oplus E$ be the $O_{Y}$-algebra whose multiplication is induced by $\phi$. Then $O_{X}$ is noncommutative, associative, and has $E$ as its trace zero elements if and only if $\phi$ has the form

$$
\begin{aligned}
\phi(z \otimes z) & =2 a^{2}+a z, & \phi(z \otimes w) & =2 a b+2 b z-a w, \\
\phi(w \otimes z) & =2 a b-b z+2 a w, & \phi(w \otimes w) & =2 b^{2}+b w,
\end{aligned}
$$

where $\{z, w\}$ is any basis for $E$, and $a$ and $b$ are elements of $O_{Y}$, not both zero.

REMARK. The above form is independent of the choice of basis for $E$. This is implied by the proposition, but can also be checked directly quite easily.

PROOF. We begin by proving the "only if" part, and assume that $\phi$ induces a noncommutative associative algebra structure on $O_{X}=O_{Y} \oplus E$, with trace zero elements $E$.

The general map $\phi$ can be written in the form

$$
\begin{aligned}
& \phi(z \otimes z)=i+a z+g w, \quad \phi(z \otimes w)=j+c z+f w, \\
& \phi(w \otimes z)=k+e z+d w, \quad \phi(w \otimes w)=l+h z+b w,
\end{aligned}
$$

where $a, b, \ldots, l$ are in $O_{Y}$. Associativity in $O_{X}$ is implied by the associativity of triple products of basis elements $\left(e_{1} e_{2}\right) e_{3}=e_{1}\left(e_{2} e_{3}\right)$ for $e_{i} \in\{z, w\}$. Therefore we must have the following equations in $O_{X}$ :

$$
\begin{aligned}
\left(z^{2}\right) z & =z\left(z^{2}\right), & & \left(w^{2}\right) w=w\left(w^{2}\right), \\
\left(z^{2}\right) w & =z(z w), & & \left(w^{2}\right) z=w(w z), \\
(z w) z & =z(w z), & & (w z) w=w(z w), \\
(w z) z & =w\left(z^{2}\right), & & (z w) w=z\left(w^{2}\right) .
\end{aligned}
$$

Using the above general form for the multiplication map $\phi$, we can compute both sides of each of these 8 equations in terms of $1, z$, and $w$ (which are a basis for $O_{X}$ ), and equate the three coefficients: This produces the explicit conditions on $a, b, \ldots, l$ for the multiplication in $O_{X}$ to be associative.

For the two equations $\left(z^{2}\right) z=z\left(z^{2}\right)$ and $\left(w^{2}\right) w=w\left(w^{2}\right)$, this gives the following six conditions: $g j=g k, g e=g c, g d=g f, h j=h k, h f=h d$, and $h e=h c$. If either $g$ or $h$ is nonzero, then $j=k, e=c$, and $d=f$ is forced, since $O_{Y}$ is an integral domain. However, this implies that $\phi(z \otimes w)=\phi(w \otimes z)$ and so the multiplication in $O_{X}$ would be commutative, contrary to assumption. Therefore, we must have

$$
g=h=0 .
$$

The other six associations produce 18 equations in the coefficients, of which 2 are identities and 2 are redundant; the remaining 14 are

$$
\begin{array}{ll}
a j=c i+f j, & b k=e k+d l, \\
j+f c=0, & k+e d=0, \\
i+a f=f^{2}, & l+b e=e^{2}, \\
c i+f k=e i+d j, & e j+d l=c k+f l, \\
j+c a+f e=k+e a+d c, & k+e f+d b=j+c d+f b, \\
e i+d k=a k, & c j+f l=b j, \\
i+a d=d^{2}, & l+b c=c^{2} .
\end{array}
$$


One can now solve for the four coefficients $i, j, k$, and $l$, obtaining

$$
i=f^{2}-a f, \quad j=-f c, \quad k=-e d, \quad l=e^{2}-b e .
$$

Upon substituting these expressions into the 10 unused equations, 2 become identities and 4 are redundant; the remaining 4 are easily factored and can be expressed as

$$
\begin{array}{ll}
(c-e)(a-f-d)=0, & (c-e)(b-e-c)=0 \\
(d-f)(a-f-d)=0, & (d-f)(b-e-c)=0 .
\end{array}
$$

Note that with these reductions, the multiplication in $O_{X}$ will be commutative if and only if $c=e$ and $d=f$; therefore, we may assume that either $c-e$ or $d-f$ is nonzero in $O_{Y}$. In either case, the above equations imply that

$$
f=a-d \text { and } e=b-c,
$$

since $O_{Y}$ is an integral domain.

This completes the analysis of the conditions imposed by associativity. The final piece of data to be used is that $E$ is the submodule of trace zero elements of $O_{X}$; this is equivalent to $\operatorname{trace}(z)=\operatorname{trace}(w)=0$, since trace is $O_{Y}$-linear. A calculation shows immediately that, in our situation, $\operatorname{trace}(z)=a+f$ and $\operatorname{trace}(w)=b+e$, so that

$$
e=-b \text { and } f=-a .
$$

Solving for the other coefficients in terms of $a$ and $b$, using (1)-(4), gives $\phi$ the form required by the proposition; moreover, $a$ and $b$ cannot both be zero $\left(O_{X}\right.$ is commutative in this case).

Conversely, it is an easy exercise to check that if $\phi$ is in that form, then $O_{X}$ is noncommutative and associative, with $E$ as the trace-zero submodule. Q.E.D.

Corollary 2. Let $O_{Y}$ be a local integral domain over $k$. Then every noncommutative $O_{Y}$-algebra $\mathcal{O}_{X}$, which is locally free of rank 3 as an $\mathcal{O}_{Y}$-module, is isomorphic to $O_{Y}\{z, w\} / I$, where $O_{Y}\{z, w\}$ is the polynomial ring over $O_{Y}$ in the noncommuting variables $z$ and $w$, and $I$ is the 2-sided ideal generated by

$$
(z+a)(z-2 a), \quad(w+b)(z-2 a), \quad(z+a)(w-2 b), \quad(w+b)(z-2 a),
$$

for some $a, b$ in $O_{Y}$, not both zero.

ProOF. When expanded, the above four equations for $I$ become exactly the 4 multiplication rules for computing $z^{2}, z w, w z$, and $w^{2}$ in terms of $1, z$, and $w$ in $O_{X}$, as given by Proposition 1. Q.E.D.

2. The global analysis. By Proposition 1 , a noncommutative $O_{Y}$-algebra $\bigcirc_{X}$, which is locally free of rank 3 over $O_{Y}$, is locally determined by two elements $a$ and $b$ in $O_{Y}$. Although, as was previously remarked, the form of the multiplication map $\phi$ does not depend on the local choice of basis for the trace zero submodule $E$, these two elements $a$ and $b$ certainly do. In order to globalize this analysis, we must describe the maps $\phi$ without resorting to any choice of basis.

Write $\phi=\phi_{1} \oplus \phi_{2}$, where $\phi_{1}: E \otimes O_{Y} E \rightarrow O_{Y}$ is the 1st coordinate of $\phi$ and $\phi_{2}: E \otimes \mathcal{O}_{Y} E \rightarrow E$ is the 2nd coordinate. Let $\underline{H}(E)$ be the submodule of 
$\underline{\text { Hom }}_{\mathcal{O}_{Y}}(E \otimes E, E)$ consisting of those maps $\phi_{2}$ which are locally of the form

$$
\begin{aligned}
\phi_{2}(z \otimes z) & =a z, & \phi_{2}(z \otimes w) & =2 b z-a w, \\
\phi_{2}(w \otimes z) & =-b z+2 a w, & \phi_{2}(w \otimes w) & =b w
\end{aligned}
$$

for some local basis $\{z, w\}$ of $E$.

Proposition 3. $\underline{H}(E)$ is canonically isomorphic to $E^{*}$.

PROOF. Define the transformation $\gamma: E^{*} \rightarrow \underline{H}(E)$ by sending a functional $\alpha: E \rightarrow \mathrm{O}_{Y}$ to the map $\phi_{2}(\alpha): E \otimes E \rightarrow E$, defined by

$$
\phi_{2}(\alpha)\left(e_{1} \otimes e_{2}\right)=2 \alpha\left(e_{2}\right) e_{1}-\alpha\left(e_{1}\right) e_{2}
$$

for $e_{1}, e_{2} \in E$. The reader can check that $\phi_{2}(\alpha)$ is indeed in $\underline{H}(E)$, and that $\gamma$ is an isomorphism, by choosing a basis $\{z, w\}$ for $E$, and using the dual basis $\left\{z^{*}, w^{*}\right\}$ of $E^{*}$. The map $\phi_{2}$ in the local form above corresponds to the functional defined by $\alpha(z)=a, \alpha(w)=b$. Q.E.D.

This description of $\underline{H}(E)$ completes the analysis of the second coordinate $\phi_{2}$ of the multiplication map $\phi$ for $O_{X}$; since the description given by Proposition 3 is independent of the choice of basis for $E$, and is natural, the local analysis sheafifies, and so in general, $\phi_{2}$ is induced from a global section of $E^{*}$.

By Proposition 1, the first coordinate $\phi_{1}$ of $\phi$ is locally determined by $\phi_{2}$. In fact, there is a global coordinate-free description of $\phi_{1}$ also.

Let $\phi_{2}$ be a global section of $\underline{H}(E)$, corresponding to a global section of $E^{*}$, or a map $\alpha: E \rightarrow O_{Y}$.

Proposition 4. With the above notation, the map $\phi_{1}: E \otimes E \rightarrow O_{Y}$ is the composition of $2(\alpha \otimes \alpha): E \otimes E \rightarrow O_{Y} \otimes O_{Y}$ with the multiplication in $O_{Y}$.

PROOF. This can be checked locally. Let $z, w$ be a local basis for $E$. If $\phi$ has the form of Proposition 1, then, as remarked during the proof of Proposition 3, its second coordinate $\phi_{2}$ corresponds to the element $a z^{*}+b w^{*}$, i.e., $\alpha$ is the map $\alpha(z)=a, \alpha(w)=b$. Therefore, $(2 \alpha \otimes \alpha)(z \otimes z)=2 a^{2},(2 \alpha \otimes \alpha)(z \otimes w)=2 a b$, $(2 \alpha \otimes \alpha)(w \otimes z)=2 b a$, and $(2 \alpha \otimes \alpha)(w \otimes w)=2 b^{2}$, which is exactly the map $\phi_{1}$. Q.E.D.

Putting these propositions together, we have the

THEOREM 5. Let $Y$ be an integral $k$-scheme. Then:

(1) Isomorphism classes of noncommutative $O_{Y}$-algebras $O_{X}$ which are locally free of rank 3 as $\mathrm{O}_{Y}$-modules are in one-to-one correspondence with isomorphism classes of pairs $(E, \alpha)$, where $E$ is a locally free rank $2 O_{Y}$-module and $\alpha: E \rightarrow O_{Y}$ is a nontrivial $O_{Y}$-linear map (or, equivalently, a global section of $\left.E^{*}\right)$.

Write $O_{X}(E, \alpha)$ for the algebra corresponding to the pair $(e, \alpha)$.

(2) $O_{X}(E, \alpha) \cong O_{Y} \oplus E$ as $O_{Y}$-modules, and $E$ corresponds to the submodule of $\mathrm{O}_{X}(E, \alpha)$ consisting of elements of trace zero. The multiplication map $\phi=$ $\phi_{1} \oplus \phi_{2}: E \otimes E \rightarrow \mathrm{O}_{Y} \oplus E$ is locally in the form of Proposition 1, for some local basis $\{z, w\}$ of $E$. Globally, the map $\phi_{2} \in \Gamma(\underline{H}(E))$ corresponds to the map $\alpha$ under the isomorphism of Proposition 3, and the map $\phi_{1}$ is $2 \alpha \otimes \alpha$.

An algebra over a $k$-scheme $Y$ restricts to an algebra over each of its closed points, i.e. an algebra over the residue fields. In this way, an $O_{Y}$-algebra can be viewed as a family of $k$-algebras parametrized by $Y$. 
PROPOSITION 6. Let $y$ be a closed point of the integral $k$-scheme $Y$, and let $\mathrm{O}_{X}(E, \alpha)$ be a noncommutative $O_{Y}$-algebra of rank 3 . Let $O_{X}(y)=O_{X}(E, \alpha) \otimes k(y)$ be the restriction of $O_{X}(E, \alpha)$ to the residue field $k(y)$ at $y$.

(1) If $\alpha \neq 0$ at $y$, then $O_{X}(y)$ is isomorphic to the algebra of $2 \times 2$ upper triangular matrices over $k(y)$.

(2) If $\alpha=0$ at $y$, then $O_{X}(y) \cong k[z, w] /\left(z^{2}, z w, w^{2}\right)$.

PROOF. We may of course work locally for these statements, and choose a basis $\{z, w\}$ for $E$ over the local ring of $Y$ at $y$. Write $\alpha(z)=a, \alpha(w)=b$; then the form of Proposition 1 applies, and we see that if $a$ and $b$ are both zero at $y$, then $z^{2}=z w=w z=w^{2}=0$, proving (2). To prove (1), we may assume $a \neq 0$ at $y$. Then the map

$$
1 \rightsquigarrow\left(\begin{array}{cc}
1 & 0 \\
0 & 1
\end{array}\right), \quad z \rightsquigarrow\left(\begin{array}{cc}
-a & 0 \\
0 & 2 a
\end{array}\right), \quad w \rightsquigarrow\left(\begin{array}{cc}
-b & 1 \\
0 & 2 b
\end{array}\right)
$$

is an isomorphism of $O_{X}(y)$ with the algebra of upper triangular $2 \times 2$ matrices over $k(y)$. Q.E.D.

That the degeneration of $\left\{\left(\begin{array}{cc}k & k \\ 0 & k\end{array}\right)\right\}$ to $k[z, w] /\left(z^{2}, z w, w^{2}\right)$ is an essentially codimension 2 phenomenon is shown by the above proposition.

3. $O_{X}(E, \alpha)$ as an algebra of endomorphisms. The aim of this final section is to globalize Proposition 6, i.e. to show that $0_{X}=O_{X}(E, \alpha)$ is an algebra of endomorphisms of a locally free rank $2 O_{Y}$-module, in fact of $E$. Let $\pi_{2}: O_{X} \rightarrow E$ be the canonical projection, and let $r: O_{X} \rightarrow \underline{\operatorname{Hom}}\left(O_{X}, O_{X}\right)$ be the regular representation of $O_{X}$. The projection $\pi_{2}$ induces a map $\pi: \underline{\operatorname{Hom}}\left(O_{X}, O_{X}\right) \rightarrow \underline{\operatorname{Hom}}\left(O_{X}, E\right)$ and the inclusion of $E$ into $O_{X}$ induces $i: \underline{\operatorname{Hom}}\left(O_{X}, E\right) \rightarrow \underline{\operatorname{Hom}}(E, E)$. Let $\beta_{1}=$ $i \circ \pi \circ r: O_{X} \rightarrow \underline{\operatorname{Hom}}(E, E)$; it is an $O_{Y}$-linear map, and locally, $\beta_{1}(x)$ sends $e \in E$ to $\pi_{2}(x e)$.

Let $\gamma: E \rightarrow \underline{\operatorname{Hom}}\left(O_{Y}, E\right)$ be the natural isomorphism, and let $\alpha^{\prime}: \underline{\operatorname{Hom}}\left(O_{Y}, E\right) \rightarrow$ $\underline{\operatorname{Hom}}(E, E)$ be the map given by composition with $\alpha: E \rightarrow O_{Y}$. Then $\beta_{2}=\alpha^{\prime} \circ \gamma \circ$ $\pi_{2}: O_{X} \rightarrow \underline{\operatorname{Hom}}(E, E)$ is $O_{Y}$-linear and, locally, $\beta_{2}(x)$ sends $e \in E$ to $\alpha(e) \cdot \pi_{2}(x)$.

Proposition 7. The $O_{Y}$-linear map $\beta=\beta_{1}+\beta_{2}: O_{X} \rightarrow \underline{\operatorname{Hom}}(E, E)$ is an $O_{Y}$-algebra monomorphism.

Proof. This can be checked locally. Choose a basis $\{z, w\}$ for $E$, and write $\alpha(z)=a, \alpha(w)=b$. By identifying $\underline{\operatorname{Hom}}(E, E)$ with $2 \times 2$ matrices over $O_{Y}$, we have

$$
\beta(1)=\left(\begin{array}{ll}
1 & 0 \\
0 & 1
\end{array}\right), \quad \beta(z)=\left(\begin{array}{cc}
2 a & 3 b \\
0 & -a
\end{array}\right), \quad \beta(w)=\left(\begin{array}{cc}
-b & 0 \\
3 a & 2 b
\end{array}\right)
$$

using the definition of $\beta(x)(e)=\pi_{2}(x e)+\alpha(e) \pi_{2}(x)$. It is an easy exercise to verify that $\beta\left(z^{2}\right)=\beta(z)^{2}, \beta(z w)=\beta(z) \beta(w), \beta(w z)=\beta(w) \beta(z)$, and $\beta\left(w^{2}\right)=\beta(w)^{2}$, which we will leave to the reader. This suffices to prove the proposition. Q.E.D.

Note that the map $\alpha$ is recovered from this representation by the composition

$$
E \rightarrow O_{X} \stackrel{\beta}{\rightarrow} \underline{\operatorname{Hom}}(E, E) \stackrel{\text { trace }}{\rightarrow} O_{Y} .
$$

The subalgebra of $\underline{\operatorname{Hom}}(E, E)$ isomorphic to $O_{X}$ consists entirely of endomorphisms which locally "factor through $\alpha$ ": 
LEMMA 8. Locally, every endomorphism $g$ in $\beta\left(O_{X}\right)$ is such that $\alpha \circ g$ factors through $\alpha$, i.e. there exists an element $t \in O_{Y}$, such that the diagram

$$
\begin{array}{lll}
E & \stackrel{\alpha}{\rightarrow} & O_{Y} \\
g \downarrow & & \downarrow_{\text {multiplication by } \mathrm{t}} \\
E & \stackrel{\alpha}{\rightarrow} & O_{Y}
\end{array}
$$

commutes.

PROOF. It suffices to check that, if $\{z, w\}$ is a local basis for $E$ over $O_{Y}$, then $\beta(1), \beta(z)$, and $\beta(w)$ satisfy the above diagram for some $t$. We leave it to the reader to check that $t=1$ works for $\beta(1), t=2 a$ works for $\beta(z)$, and $t=2 b$ works for $\beta(w)$. Q.E.D.

By Lemma 8, any endomorphism in $\beta\left(O_{X}\right)$ must preserve the kernel of $\alpha$, which is a rank 1 subsheaf of $E$; at points where $\alpha \neq 0$, a basis for $E$ may be extended from a generator for this kernel, and the elements of $\beta\left(O_{X}\right)$ will be represented by upper triangular matrices in this basis. However, it is not true that $\beta\left(O_{X}\right)$ consists of all such matrices, i.e. $\beta\left(O_{X}\right)$ is not the algebra of all $g$ in $\operatorname{End}(E)$ which locally satisfy Lemma 8. Our algebra $\beta\left(O_{X}\right)$ is only the algebra of $g$ 's which "obviously" satisfy the commutative diagram. Let us be more precise: Motivated by the construction of the Koszul complex, we make the following

DEFINITION 9. Let $E$ be a locally free rank $2 O_{Y}$-module and $\alpha: E \rightarrow O_{Y}$ a nontrivial $O_{Y}$-linear map. The Koszul algebra of $(E, \alpha)$, denoted by $K(E, \alpha)$ is the subalgebra of End $(E)$ generated (locally) by the matrices

$$
\left(\begin{array}{ll}
1 & 0 \\
0 & 1
\end{array}\right), \quad\left(\begin{array}{ll}
a & b \\
0 & 0
\end{array}\right), \quad\left(\begin{array}{ll}
0 & 0 \\
a & b
\end{array}\right)
$$

with respect to some local basis $\{z, w\}$ of $E$, where $\alpha(z)=a, \alpha(w)=b$.

It can be easily checked that $K(e, \alpha)$ is locally free of rank 3 as an $O_{Y}$-module, and that it is a subalgebra of $\underline{\operatorname{End}}(E)$. Moreover, every element of $K(E, \alpha)$ locally satisfies Lemma 8, and the algebra is independent of the local choice of basis for $E$.

THEOREM 10. The map $\beta$ is an isomorphism of $\mathcal{O}_{X}(E, \alpha)$ onto the Koszul algebra $K(E, \alpha)$.

ProOF. This can be checked locally; choose a basis $\{z, w\}$ for $E$. Then

$$
\beta(z)=\left(\begin{array}{cc}
2 a & 3 b \\
0 & -a
\end{array}\right)=-a\left(\begin{array}{ll}
1 & 0 \\
0 & 1
\end{array}\right)+3\left(\begin{array}{ll}
a & b \\
0 & 0
\end{array}\right),
$$

and similarly for $\beta(w)$, showing that $\beta$ maps $O_{X}(E, \alpha)$ into $K(E, \alpha)$. On the other hand, $\left(\begin{array}{ll}a & b \\ 0 & 0\end{array}\right)=\frac{1}{3}[\beta(z)+a \beta(1)]$ and similarly for $\left(\begin{array}{ll}0 & 0 \\ a & b\end{array}\right)$ which proves surjectivity. Q.E.D.

It may seem that we have defined our way out of identifying the algebra $O_{X}(E, \alpha)$ by the above, and that is a fair criticism. To be complete, we should answer the following question: How far is the Koszul algebra from the full subalgebra of End $(E)$ consisting of elements which locally satisfy Lemma 8? Let us denote this algebra by $\tilde{O}_{X}(E, \alpha)$ and let $\underline{C}$ be the cokernel of the inclusion of $\mathcal{O}_{X}(E, \alpha) \cong K(E, \alpha)$ into $\tilde{O}_{X}(E, \alpha)$. Our answer to the above question is to identify the algebra $\tilde{O}_{X}(E, \alpha)$. 
Proposition 11. (1) The sheaf $\underline{C}$ is supported on the zero locus $Z$ of $\alpha$ (whose ideal sheaf is $\left.\alpha(E) \subset O_{Y}\right)$.

(2) Let $D$ be the divisor of zeroes of $\alpha$, and write $E(D)$ for $E \otimes O_{Y}(D)$. Note that $\alpha$ factors through $\bigcirc_{Y}(-D)$, thereby inducing a map $\alpha(D): E(D) \rightarrow O_{Y}$. Then $\tilde{O}_{X}(E, \alpha) \cong \tilde{O}_{X}(E(D), \alpha(D))$.

(3) If $Y$ is factorial and $D$ is zero, then $\underline{C}$ is zero and $\tilde{\mathcal{O}}_{X}(E, \alpha) \cong \mathcal{O}_{X}(E, \alpha)$.

(4) If $Y$ is factorial, then $\tilde{O}_{X}(E, \alpha) \cong \mathcal{O}_{X}(E(D), \alpha(D))$.

PROOF. To prove (1), we may work locally, and choose a basis $\{z, w\}$ for $E$; moreover, if we assume $\alpha \neq 0$ at $y \in Y$, then we may assume $a \neq 0$ at $y$, so that $a$ is a unit in the local ring $O_{Y, y}$ at $y$, and by replacing $z$ by $a^{-1} z$ we may assume $a=1$. In this case $K(E, \alpha)$ is generated by the matrices $\left(\begin{array}{ll}1 & 0 \\ 0 & 1\end{array}\right),\left(\begin{array}{ll}1 & b \\ c & 0\end{array}\right)$, and $\left(\begin{array}{ll}0 & 0 \\ 1 & b\end{array}\right)$. Assume finally that $\left(\begin{array}{ll}p & q \\ u & v\end{array}\right)$ is in $\tilde{O}_{X}(E, \alpha)$. Hence there is a $t \in O_{Y, y}$ such that $p+b u=t$ and $q+b v=t b$. Therefore,

$$
\left(\begin{array}{ll}
p & q \\
u & v
\end{array}\right)=(v-u b)\left(\begin{array}{ll}
1 & 0 \\
0 & 1
\end{array}\right)+(t-v)\left(\begin{array}{ll}
1 & b \\
0 & 0
\end{array}\right)+u\left(\begin{array}{ll}
0 & 0 \\
1 & b
\end{array}\right)
$$

and is in $K(E, \alpha)$, proving (1).

To prove (2), consider the map $\bar{\alpha}: E \rightarrow O_{Y}(-D)$ (which is $\alpha$ !), and note that for an endomorphism $g$ of $E, \alpha \circ g$ factors through $\alpha$ if and only if $\bar{\alpha} \circ g$ factors through $\bar{\alpha}$. In addition, the algebra of endomorphisms of $E$ factoring through $\bar{\alpha}$ is isomorphic to the algebra of endomorphisms of $E(D)$ factoring through $\alpha(D)$; the isomorphism is obtained by twisting the maps by $O_{Y}(D)$. Hence $\tilde{O}_{X}(E, \alpha)$ is isomorphic to $\tilde{O}_{X}(E(D), \alpha(D))$.

For the rest, assume $Y$ is factorial. Working locally, if $D=0$ then, when one factors $a$ and $b$ into irreducibles, there can be no common factors. Moreover, since

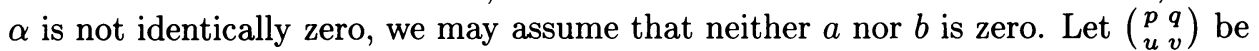
in $\tilde{O}_{X}(E, \alpha)$. Then there exists $t$ in $\mathcal{O}_{Y}$ such that $a p+b u=a t$ and $a q+b v=b t$. Therefore, $a \mid b u$ and $b \mid a q$, and since $D=0, a \mid u$ and $b \mid q$. Write $u=a r$ and $q=b s$; then $p+b r=t$ and $a s+v=t$, so that $p-a s=v-b r$. Call this element $x$; then

$$
\left(\begin{array}{ll}
p & q \\
u & v
\end{array}\right)=x\left(\begin{array}{ll}
1 & 0 \\
0 & 1
\end{array}\right)+s\left(\begin{array}{ll}
a & b \\
0 & 0
\end{array}\right)+r\left(\begin{array}{ll}
0 & 0 \\
a & b
\end{array}\right),
$$

and is therefore in $K(E, \alpha) \cong \mathrm{O}_{X}(E, \alpha)$. Thus $\underline{C}=0$, proving (3).

Statement (4) follows from applying (3) to the pair $(E(D), \alpha(D))$; by construction, the divisor of zeroes of $\alpha(D)$ is zero, so that

$$
\tilde{O}_{X}(E(D), \alpha(D)) \cong \mathcal{O}_{X}(E(D), \alpha(D)) \text {. }
$$

By $(2), \tilde{O}_{X}(E, \alpha) \cong \tilde{O}_{X}(E(D), \alpha(D))$ in any case; combining these isomorphisms proves (4). Q.E.D.

Finally, let us address the following situation. Suppose $L$ is a locally free rank 1 subsheaf of a locally free rank 2 sheaf $F$. Let $A$ be the sheaf of algebras of endomorphisms of $F$ preserving $L$. The above proposition allows us to determine $A$ in the case where $Y$ is factorial, as follows. Let $D$ be the divisor of zeroes of the inclusion $i: L \rightarrow F$. By twisting and dualizing, $i$ corresponds to a map $\alpha: F^{*} \otimes$ $L \rightarrow O_{Y}$ whose divisor of zeroes is also $D$; moreover, $A$ is naturally isomorphic to $\tilde{\mathcal{O}}_{X}\left(F^{*} \otimes L, \alpha\right)$. Therefore by Proposition 11, (4), $A \cong \mathcal{O}_{X}\left(F^{*} \otimes L \otimes \mathcal{O}_{Y}(D), \alpha(D)\right)$. 
ACKNOWLEDGEMENT. The authors would like to thank Lawrence Ein for several helpful suggestions during the revision of this paper.

\section{REFERENCES}

1. R. Miranda, Triple covers in algebraic geometry, Amer. J. Math. (to appear).

Department of Mathematics, Tufts University, Medford, Massachusetts 02155

Department of Mathematics, Bar-Ilan University, Ramat-Gan, ISRAel (Current address of Mina Teicher)

Current address: (Rick Miranda): Department of Mathematics, Colorado State University, Fort Collins, Colorado 80523 